\title{
ASOCIACIÓN ENTRE ENDOMETRIOSIS PÉLVICA Y ESTRÉS OXIDATIVO
}

\section{Association between pelvic endometriosis and oxidative stress}

\author{
Johana Márquez-Lázaro, MSc(c) ${ }^{1}$; Maricela Viola-Rhenals, $\mathrm{PhD}^{2}$; \\ Álvaro Monterrosa-Castro, $\mathrm{MD}^{3}$ \\ Recibido: octubre 22/12 - Aceptado: mayo 29/13
}

\section{RESUMEN}

Objetivo: precisar el estado actual del conocimiento referente a la asociación entre endometriosis pélvica y estrés oxidativo.

Materiales y métodos: se realizó búsqueda temática en las bases de datos Sciencedirect y Medline vía Pubmed. Se seleccionaron estudios publicados en lengua inglesa entre enero de 1990 y junio del 2012, que hubieran evaluado la asociación entre estrés oxidativo y endometriosis pélvica.

Resultados: de 984 resúmenes identificados, 201 fueron escogidos por tener información relacionada; 110 artículos completos fueron evaluados, 45 que tenían información relevante acorde con el objetivo de la revisión fueron tenidos en cuenta. Existen estudios en humanos y en modelos experimentales que proponen que el estrés oxidativo puede estar involucrado en la fisiopatología de la endometriosis pélvica. Distintos estudios señalan que las mujeres

1 Química, candidata a Magíster en bioquímica, Facultad de Medicina, Universidad de Cartagena. Cartagena, Colombia.

2 Química farmacéutica. Doctor en Ciencias mención Bioquímica. Docente, directora Grupo de Investigación bioquímica y biología celular del cáncer, Facultad de Medicina, Universidad de Cartagena. Cartagena, Colombia.

3 Médico, Especialista en Ginecología y Obstetricia. Docente, director Grupo de Investigación Salud de la mujer, Facultad de Medicina, Universidad de Cartagena. Cartagena, Colombia.

alvaromonterrosa@gmail.com con infertilidad asociada a endometriosis presentan niveles más altos de prooxidantes y más bajos de antioxidantes, con respecto al control sano, en mediciones en líquido peritoneal o tejido endometrial, sin consistencia con los hallazgos en plasma o suero. Conclusiones: no es claro el papel que desempeñan las especies reactivas de oxígeno en la endometriosis pélvica. Este campo se presenta como un atractivo ámbito de investigación en ciencias básicas y aplicadas.

Palabras clave: endometriosis, estrés oxidativo, infertilidad, especies reactivas de oxígeno.

\section{ABSTRACT}

Objective: To determine the current state of knowledge regarding the association between pelvic endometriosis and oxidative stress.

Materials and methods: A search on the topic was conducted in the Sciencedirect and Medline databases through Pubmed. Studies published in the English language between January 1990 and June 2012 assessing the association between oxidative stress and pelvic endometriosis were selected.

Results: Of a total of 984 abstracts identified, 201 were selected because they contained related information. Overall, 110 complete articles were assessed and, of them, 45 that contained relevant information consistent with the objective of the 
review were taken into account. There are studies in experimental models and in humans suggesting the potential involvement of oxidative stress in the pathophysiology of pelvic endometriosis. Different studies point to the fact that women with endometriosis-associated infertility have higher levels of pro-oxidants and lower levels of antioxidants when compared with healthy controls, as measured in peritoneal fluid or endometrial tissue; these levels are not consistent plasma or serum levels.

Conclusions: The role of reactive oxygen species in pelvic endometriosis is yet unclear. This field opens an interesting possibility for research in basic and applied science.

Key words: Endometriosis, oxidative stress, infertility, reactive oxygen species.

\section{INTRODUCCIÓN}

La endometriosis consiste en la aparición y crecimiento de tejido endometrial fuera del útero, más frecuentemente en la cavidad pélvica, ovarios, ligamentos uterinos, vejiga urinaria, intestino, entre otros. Su prevalencia varía entre 6 y un $10 \%$ de la población femenina y afecta a más del $50 \%$ de las mujeres de parejas infértiles (1). Es una de las entidades ginecológicas que más frecuentemente se presenta en la etapa reproductiva (2). Las causas de la endometriosis pélvica no han sido esclarecidas, aunque existen varias teorías que intentan explicar el fenómeno, sin que ninguna de ellas lo logre del todo, las principales son: a) el trasplante ectópico del tejido endometrial por medio de la menstruación retrógrada, b) la metaplasia celómica con transformación del epitelio peritoneal en endometrio, y c) la teoría de la inducción que propone la participación de uno o varios factores endógenos, bioquímicos o inmunológicos (2).

Se consideran factores de riesgo: madre o hermana con endometriosis, menarquia a edades tempranas, ciclos menstruales cortos y menstruaciones superiores a siete días. Puede no tener manifestaciones clínicas o puede causar sintomatología que varía desde el dolor pélvico cíclico y crónico, las menstruaciones dolorosas que causan incapacidad, los episodios prolongados de metrorragias o la dispareunia intensa o infertilidad $(1,3)$. No existe relación entre la severidad de la endometriosis y la intensidad o presencia de las manifestaciones (1).

Respecto al estrés oxidativo, este se ha definido como el desbalance entre antioxidantes y especies reactivas de oxígeno, que alteran estructuras celulares con cambios en proteínas, lípidos, ADN y carbohidratos $(4,5)$. Son fuentes de estrés oxidativo: a) la cadena respiratoria y la fosforilación oxidativa mitocondrial, b) la exposición a la radiación ionizante, c) el metabolismo de compuestos exógenos, y d) algunos procesos patológicos como el envejecimiento, la enfermedad de Alzheimer, el cáncer e incluso la inflamación, entre otros $(5,6)$.

Las especies reactivas de oxígeno -en inglés reactive oxygen species (ROS) - son un grupo de moléculas que pueden ser agentes oxidantes o ser fácilmente convertidos en ellos, pueden ser divididas en: radicales libres, especies químicas capaces de existir independientemente y contienen uno o más electrones desapareados, como el radical hidroxilo $(\mathrm{OH})$, el anión hidroxilo $(-\mathrm{OH})$ y el anión superóxido $\left(\mathrm{O}_{2}^{-}\right)$, y especies no radicales, moléculas con una fuerte capacidad oxidante como el peróxido de hidrógeno $\left(\mathrm{H}_{2} \mathrm{O}_{2}\right)$, el ozono $\left(\mathrm{O}_{3}\right)$ y el oxígeno singlete $\left({ }^{1} \mathrm{O}_{2}\right)$ (6). Cuando los radicales libres reaccionan con los no radicales, el resultado es un nuevo radical que inicia una reacción en cadena de más radicales libres.

El anión superóxido y el peróxido de hidrógeno no son los más tóxicos, aunque poseen una vida media larga, la toxicidad depende de la facilidad con la que son convertidos en radicales hidroxilo, siendo este último el de mayor toxicidad. In vivo, la producción de la mayoría de los radicales hidroxilos se debe a las reacciones acopladas de Fenton y Haber-Weiss las cuales son catalizadas por metales $(7,8)$.

Debido a los efectos dañinos de las ROS, los organismos aeróbicos han desarrollado diferentes 
mecanismos de defensa para mantener la estabilidad celular, que pueden clasificarse en enzimáticos y no enzimáticos, los cuales se interrelacionan y actúan en conjunto para proteger a las células de la toxicidad de las $\operatorname{ROS}(6,9,10)$.

Las enzimas antioxidantes. Superóxido dismutasa (SOD), catalasa (CAT) y glutatión peroxidasa (GPx) son el esqueleto del sistema de defensa antioxidante celular. El SOD cataliza la dismutación del anión radical superóxido a peróxido de hidrógeno y tiene tres isoformas: $\mathrm{MnSOD}, \mathrm{Cu} / \mathrm{ZnSOD}$ y EcSOD, las cuales poseen diferentes iones metálicos como cofactores (10-12). La CAT es una enzima tetramérica que detoxifica peróxido de hidrógeno para producir agua y oxígeno molecular, está compuesta por cuatro subunidades idénticas de $60 \mathrm{KDa}$, con cuatro grupos ferriprotoporfirinas por molécula (11). El GPx reduce tanto peróxidos como hidroperóxidos orgánicos usando al glutatión reducido (GSH). Existen por lo menos cinco isoformas de GPx encontradas en mamíferos, tienen un peso aproximado de $80 \mathrm{KDa}$ y contienen selenocisteína en cada una de las cuatro subunidades, esencial para la actividad de la enzima. En ausencia de estrés oxidativo las ROS se mantienen en bajas concentraciones por la acción coordinada de estas tres enzimas $(11,12)$. Los antioxidantes no enzimáticos extracelulares e intracelulares son también una importante línea de defensa frente a los ROS. Algunas moléculas antioxidantes no enzimáticas son: la bilirrubina, el glutatión reducido, el ácido úrico y otras moléculas con grupos sulfhídricos en su estructura, las vitaminas A, C y E $(3,13,14)$.

Las células contienen sensores redox que son sustancias capaces de detectar cambios en el potencial de membrana debido a la presencia de ROS. Esos sensores hacen parte del complejo sistema de señalización celular. Las cascadas de señalización activadas por estrés oxidativo pueden ser alteradas por genotóxicos endógenos como radiación, presencia de citoquinas inflamatorias y algunos compuestos químicos. A su vez, las ROS pueden alterar las cascadas de transducción de señales, como también inducir cambios en factores de transcripción que median la respuesta inmediata al estrés celular oxidativo (15).

Estrés oxidativo y endometriosis. La mujer con endometriosis pélvica presenta un proceso inflamatorio que produce la activación de macrófagos en la cavidad peritoneal. Desde el punto de vista teórico, ese evento potencialmente llevaría a estrés oxidativo, trayendo como consecuencia oxidación de proteínas y peroxidación de lípidos. La activación masiva de polimorfos nucleares también aumentaría el estrés oxidativo mediado por el aumento de las ROS, con daños en las membranas de los glóbulos rojos, las células endometriales y peritoneales $(3,6,8)$, todo ello facilitaría la liberación de hemoglobina la cual, al degradarse, dejaría libre, entre otros productos, el hierro, que a su vez puede catalizar la conversión del $\mathrm{H}_{2} \mathrm{O}_{2}$ al radical hidroxilo $\left(\mathrm{OH}^{-}\right)$, que es una ROS altamente reactiva, posiblemente por un mecanismo de reacciones tipo Fenton, que a su vez contribuye a prolongar el daño oxidativo $(7,8,16)$.

Recientemente se ha implicado al estrés oxidativo en la patogénesis de la endometriosis. Se ha evidenciado que en la endometriosis pélvica las ROS pueden provenir de los eritrocitos, el tejido endometrial apoptótico y los fagocitos mononucleares resultantes de la menstruación retrograda, lo que trae consigo el daño a macromoléculas y la consecuente formación de peróxidos lipídicos, proteínas oxidadas y daños en el ácido desoxirribonucleico $(\mathrm{ADN})(13,14)$.

No obstante el conocimiento y estudio detallado de esas reacciones bioquímicas, son pocos los estudios que describen el papel del estrés oxidativo en el origen o en el agravamiento de la endometriosis pélvica. Son múltiples los indicadores utilizados, diversos los tejidos evaluados, sin embargo, los datos publicados aún no son concluyentes y no es claro si la endometriosis incrementa el estrés oxidativo o si podría surgir como consecuencia del incremento del mismo. Por tanto, el objetivo de esta revisión es precisar el conocimiento actual sobre la asociación entre endometriosis pélvica y estrés oxidativo. 


\section{MATERIALES Y MÉTODOS}

Se incluyeron estudios primarios sin restricción por diseño, además de revisiones temáticas o sistemáticas. Se consideraron estudios realizados con sangre, líquido peritoneal, tejido endometrial o fluido folicular, en donde se evaluaron marcadores de estrés oxidativo como peroxidación lipídica, actividad de enzimas antioxidantes, niveles de antioxidantes endógenos y niveles de especies reactivas de oxígeno. Se incluyeron estudios en humanos, modelos animales y líneas celulares. El tipo de asociación fue estrés oxidativo con endometriosis pélvica.

Se realizó la búsqueda en las bases de datos Science Direct y Medline vía Pubmed. La búsqueda se limitó al idioma inglés y a publicaciones realizadas entre enero de 1990 y junio del 2012. Las palabras claves empleadas para la búsqueda fueron: "endometriosis", "oxidative stress", "reactive oxygen species" e "infertility".

Se revisaron los títulos y los resúmenes obtenidos a partir de la búsqueda en las bases de datos, se escogieron aquellos que cumplieron con los criterios de inclusión. Se eliminaron las publicaciones duplicadas.

Análisis. Los resultados fueron presentados de forma cualitativa y cuantitativa mediante los estimadores epidemiológicos o estadísticos de asociación realizados en los estudios considerados. No se evaluó alguna intervención en los estudios revisados.

\section{RESULTADOS}

Se obtuvieron 984 resúmenes de artículos; 201 $(20,4 \%)$ resúmenes fueron escogidos por tener información relacionada, 110 (54,7\%) estuvieron disponibles en texto completo y fueron revisados. De estos se consideró que 45 (40,9\%) incluían información relevante y fueron los tenidos en cuenta para la revisión (figura 1).

En los últimos años se ha ido incrementando el número de investigaciones que tratan de establecer la importancia de la asociación entre endometriosis y estrés oxidativo. Estos trabajos generalmente

Figura 1.

Flujograma de búsqueda bibliográfica

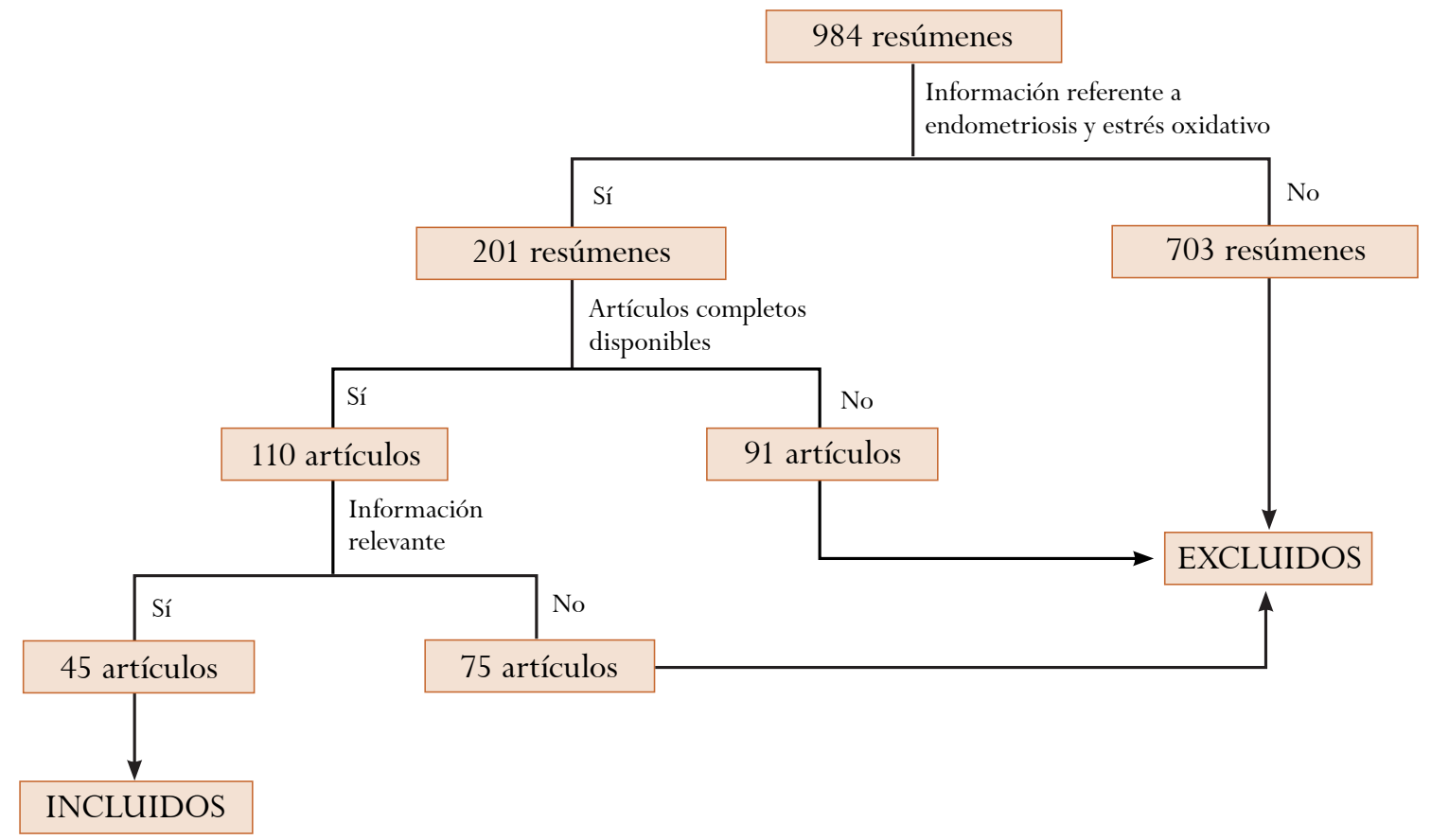


emplean muestras de sangre, fluido folicular, tejido endometrial o líquido peritoneal, evaluando en ellas peroxidación lipídica y actividad de enzimas antioxidantes como por ejemplo catalasa (CAT), superóxido dismutasa (SOD), niveles de 8-hidroxi2-deoxiguanosina, glutatión peroxidasa (GPx), e incluso niveles de glutatión (GSH) y vitamina E, los cuales son antioxidantes no enzimáticos. La tabla 1 presenta 32 de los estudios que fueron considerados como de mayor relevancia, precisando el tejido o fluido valorado, los marcadores utilizados y los resultados cualitativa y cuantitativamente encontrados.

Uno de los marcadores más estudiados es la concentración de vitamina E. Los resultados encontrados no son consistentes. Seeber et al. (13) muestran que no hay diferencias entre los niveles de vitamina E en muestras de líquido peritoneal en mujeres con endometriosis y mujeres sin endometriosis. Por otra parte, otros autores señalan que hay altos niveles de ese marcador en suero y líquido peritoneal en mujeres que presentan endometriosis $(17,18)$, y se ha señalado que la administración de vitamina E puede llegar a revertir el proceso fisiopatológico de la entidad, demostrando de paso el papel importante de la oxidación en el daño estructural peritoneal (19).

Otra importante consideración es la referente al daño estructural con la liberación de los lípidos, los cuales a su vez pueden ser cuantificados como malonildialdehído (MDA), que ha sido estudiado en suero, líquido peritoneal y fluido folicular. Las conclusiones planteadas son diversa, van desde la ausencia de diferencias significativas entre mujeres con endometriosis frente a mujeres libres de endometriosis, hasta el aumento del marcador en mujeres con endometriosis pélvica (18, 20-24). Situaciones similares se observan al abordar estudios que consideraron otros marcadores como SOD o CAT $(16,25-28)$.

$\mathrm{Al}$ evaluar en modelos animales con endometriosis la acción de la melatonina y la curcumina se encontró que estos compuestos disminuyen la progresión de la enfermedad. La melatonina aumenta los niveles de actividad de CAT y SOD, y genera disminución de MDA y de curcumina, con reducción de la expresión de MMP-3 y la translocación de NF-kB al núcleo $(29,30)$.

Existen grandes intentos por entender los mecanismos de establecimiento y desarrollo de la enfermedad, y la manera como el estrés oxidativo interviene en el proceso. Para ello, además de estudiar los fluidos y tejidos humanos, así como modelos en especies animales inferiores, hay investigaciones realizadas en cultivos primarios de células estromales y epiteliales, donde se ha observado que al agregar a los ambientes celulares sustancias con acciones antioxidantes se puede llegar a inhibir la proliferación celular, siendo este un mecanismo de acción en el que se involucra el estrés oxidativo $(27,29,31)$.

\section{DISCUSIÓN}

Aunque varios estudios evidencian la posible asociación entre el estrés oxidativo y la endometriosis, ninguno de ellos es concluyente (32-47). La ausencia de una respuesta uniforme puede guardar relación en parte con la heterogeneidad de los diversos estudios. Diferentes variables consideradas impiden las miradas en conjunto, o intentar unificar los resultados. Existen sensibles diferencias en el tipo de muestra que se consideran, las edades de las pacientes, el grupo control empleado, los factores genéticos y los étnicos asociados, entre otros. Usualmente es solo uno o son pocos los marcadores estudiados cuando se amerita. Para que los resultados obtenidos sean relevantes, se debe adelantar la evaluación con el mayor número de marcadores de estrés oxidativo en la misma muestra y bajo los mismos parámetros de intervención.

La presente revisión tiene como limitaciones ser temática en una muestra de estudios por conveniencia y sin realizar intervención en los mismos, incluyendo estudios de diferentes niveles de evidencia y sin precisar sobre el correcto o no diseño metodológico. No obstante, permite observar la diversidad de marcadores que han sido propuestos y evaluados, 


\begin{tabular}{|c|c|c|c|c|}
\hline Autor & $\begin{array}{l}\text { Tipo de } \\
\text { muestra }\end{array}$ & Marcador & Hallazgo cualitativo & Hallazgo cuantitativo \\
\hline $\begin{array}{l}\text { Seeber } \text { et al., } \\
2010 \text { (13) }\end{array}$ & $\begin{array}{c}\text { Suero } \\
\text { líquido } \\
\text { peritoneal }\end{array}$ & $\begin{array}{l}\text { Niveles de } \\
\text { afamina y } \\
\text { vitamina } E\end{array}$ & $\begin{array}{l}\text { Niveles de afamina en líquido } \\
\text { peritoneal están correlacionados } \\
\text { con los niveles de vitamina } \mathrm{E} \text {. } \\
\text { No hay diferencias en los niveles } \\
\text { de vitamina E unida a afamina en } \\
\text { las muestras de líquido peritoneal. } \\
\text { Niveles en suero de afamina y } \\
\text { vitamina E fueron similares en los } \\
\text { grupos estudiados. }\end{array}$ & $\begin{array}{c}\text { Afamina en líquido peritoneal } \\
\text { Control }=25,87 \pm 1,41 \mathrm{mg} / \mu \mathrm{L} \\
\text { Endometriosis }=31,91 \pm 1,8 \mathrm{mg} / \mu \mathrm{L} \\
p=0,012 \\
\text { Correlación entre niveles de } \\
\text { afamina y vitamina E: } \mathrm{r}=0,635 \\
p<0,0001\end{array}$ \\
\hline $\begin{array}{l}\text { Savaris et al., } \\
2011 \text { (14) }\end{array}$ & Suero & $\begin{array}{l}\text { Capacidad } \\
\text { antioxidante } \\
\text { (niveles de } \\
\text { tioles) }\end{array}$ & $\begin{array}{l}\text { La capacidad antioxidante fue más } \\
\text { baja en mujeres con endometriosis. }\end{array}$ & $\begin{array}{c}\text { Tioles no proteicos: } \\
\text { Control }=372,6 \pm 216,76 \mu \mathrm{M} \\
\text { Endometriosis }=180,98 \pm 99,78 \mu \mathrm{M} \\
\qquad p=0,001\end{array}$ \\
\hline $\begin{array}{l}\text { Azevedo et al., } \\
2010 \text { (38) }\end{array}$ & $\begin{array}{c}\text { Tejido } \\
\text { endometrial } \\
\text { eutópico }\end{array}$ & $\begin{array}{c}\text { Anión } \\
\text { superóxido } \\
\text { (tinción con } \\
\text { dihidroetidium) }\end{array}$ & $\begin{array}{l}\text { Las imágenes mostraron mayor } \\
\text { tinción de dihidroetidium en las } \\
\text { muestras de las mujeres con } \\
\text { endometriosis vs control. }\end{array}$ & No reportado \\
\hline $\begin{array}{l}\text { Campos et al., } \\
2008 \text { (18) }\end{array}$ & $\begin{array}{l}\text { Suero } \\
\text { fluido folicular }\end{array}$ & $\begin{array}{l}\text { Peroxidación } \\
\text { lipídica como } \\
\text { malonildial- } \\
\text { dehído (MDA). } \\
\text { Niveles de } \\
\text { vitamina E }\end{array}$ & $\begin{array}{l}\text { No se encontraron diferencias } \\
\text { significativas entre los niveles } \\
\text { de MDA en suero y fluido folicular } \\
\text { de las mujeres con endometriosis } \\
\text { vs el control. }\end{array}$ & $\begin{array}{c}\text { MDA en Control }=1,07 \\
\mathrm{nmol} / \mathrm{mL} \text { Endometriosis }=1,64 \mathrm{nmol} / \mathrm{mL} \\
p>0,05 \\
\text { Vitamina } \mathrm{E} \text { en Control }=6,88 \mu \mathrm{mol} / \mathrm{L} \\
\text { Endometriosis }=5,59 \mu \mathrm{mol} / \mathrm{L} \\
p>0,05\end{array}$ \\
\hline $\begin{array}{l}\text { Szczepanska et al., } \\
2003 \text { (20) }\end{array}$ & $\begin{array}{c}\text { Líquido } \\
\text { peritoneal } \\
\text { dismutasa (SOD) } \\
\text { y glutatión } \\
\text { peroxidasa (Gpx). } \\
\text { Estado } \\
\text { antioxidante total. } \\
\text { Niveles de per } \\
\text { oxidaciónlipídica } \\
\text { (MDA) }\end{array}$ & $\begin{array}{l}\text { Actividad } \\
\text { superóxido }\end{array}$ & $\begin{array}{l}\text { La actividad superóxido dismutasa y } \\
\text { glutatión peroxidasa fue menor en } \\
\text { mujeres con endometriosis. } \\
\text { El estado total antioxidante fue más bajo } \\
\text { en las mujeres con endometriosis. } \\
\text { Los niveles de peroxidación lipídica fueron } \\
\text { más altos en las mujeres con endometriosis. }\end{array}$ & $\begin{array}{c}\text { SOD en Control }=1,921 \pm 0,175 \mathrm{U} / \mathrm{mL} \\
\text { Endometrios }=1,030 \pm 0,176 \mathrm{U} / \mathrm{mL} \\
\text { Gpx en Control }=1122,375 \pm 96,27 \mathrm{ng} / \mathrm{mL} \\
\text { Endometriosis }=870,21 \pm 52,17 \mathrm{ng} / \mathrm{mL} \\
\text { Peroxidación lipídica } \\
\text { Control }=1,075 \pm 0,305 \mathrm{mmo} / \mathrm{L} \\
\text { Endometriosis }=0,505 \pm 0,212 \mathrm{mmol} / \mathrm{L} \\
\text { MDA en Control }=15,578 \pm 35,66 \mu \mathrm{M} \\
\text { Endometriosis }=23,074 \pm 22,12 \mu \mathrm{M}\end{array}$ \\
\hline $\begin{array}{l}\text { Langendonckt } \\
\text { et al., } 2002 \text { (4) }\end{array}$ & $\begin{array}{l}\text { Sangre } \\
\text { líquido } \\
\text { peritoneal, } \\
\text { tejido } \\
\text { endometrial }\end{array}$ & $\begin{array}{l}\text { Niveles de hierro } \\
\text { y ferritina. } \\
\text { Tinción de } \\
\text { depósitos de } \\
\text { hierro en tejido } \\
\text { endometrial }\end{array}$ & $\begin{array}{l}\text { Los niveles de hierro fueron más bajos en } \\
\text { el líquido peritoneal que en sangre, en cuanto } \\
\text { a las concentraciones de ferritina estas fueron } \\
\text { mayores en líquido peritoneal que en sangre. } \\
\text { Depósitos de hierro fueron encontrados } \\
\text { en las células estromales del tejido } \\
\text { endometrial eutópico y ectópico. }\end{array}$ & $\begin{array}{l}\text { Niveles de ferritina en líquido peritoneal } \\
5,2 \pm 3,9 \text { vs } 4,5 \pm 2,2 \text { en suero }\end{array}$ \\
\hline $\begin{array}{l}\text { Louusse et al., } \\
2009 \text { (16) }\end{array}$ & $\begin{array}{c}\text { Líquido } \\
\text { peritoneal } \\
\text { peritoneales. }\end{array}$ & $\begin{array}{l}\text { Niveles de ferritina } \\
\text { en macrófagos } \\
\text { Niveles de } \\
\text { transferina, ferritina } \\
\text { y prohepcidina en } \\
\text { líquido peritoneal }\end{array}$ & $\begin{array}{l}\text { La densidad de macrófagos peritoneales fue } \\
\text { significativamente alta en mujeres con } \\
\text { endometriosis vs controles. } \\
\text { El líquido peritoneal de mujeres con } \\
\text { endometriosis presentó niveles } \\
\text { significativamente altos de saturaciones de } \\
\text { transferrina y ferritina vs control. } \\
\text { No se encontró diferencia entre los niveles } \\
\text { de prohepcidina en líquido peritoneal. }\end{array}$ & $\begin{array}{l}\text { Niveles de saturaciones de ferritina, } \\
\qquad p=0,048\end{array}$ \\
\hline
\end{tabular}




\begin{tabular}{|c|c|c|c|c|}
\hline Autor & $\begin{array}{l}\text { Tipo de } \\
\text { muestra }\end{array}$ & Marcador & Hallazgo cualitativo & Hallazgo cuantitativo \\
\hline $\begin{array}{l}\text { Mier-Cabrera } \\
\text { et al., } 2008(21)\end{array}$ & $\begin{array}{l}\text { Plasma } \\
\text { líquido peritoneal } \\
\text { lipídicos }\end{array}$ & $\begin{array}{l}\text { Malonildialdehído } \\
\qquad \text { (MDA) } \\
\text { Hidroperóxidos }\end{array}$ & $\begin{array}{l}\text { Los niveles de hidroperóxidos lipídicos y MDA } \\
\text { disminuyeron al suplementar con vitamina } \\
\text { C y E la dieta de mujeres con endometriosis, } \\
\text { después de pasados } 4 \text { a } 6 \text { meses del } \\
\text { tratamiento vs grupo con placebo. }\end{array}$ & $\begin{array}{c}\text { Niveles bajos de MDA de } 4 \text { a } 6 \text { meses } \\
\qquad<0,05 \\
\text { Niveles de hidroperóxidos lipídicos } \\
p<0,05\end{array}$ \\
\hline $\begin{array}{l}\text { Foyouzi et al., } \\
2004 \text { (31) }\end{array}$ & $\begin{array}{l}\text { Cultivo celular } \\
\text { primario de } \\
\text { biopsias de tejido } \\
\text { endometrial de } \\
\text { pacientes con y } \\
\text { sin endometriosis }\end{array}$ & $\begin{array}{l}\text { Proliferación de } \\
\text { células estromales. } \\
\text { Ensayo de MTT. }\end{array}$ & $\begin{array}{l}\text { Se encontró que los antioxidantes: succinato } \\
\text { de vitamina E, Ebselen y } \mathrm{N} \text {-acetil cisteína } \\
\text { inhiben la proliferación celular de células } \\
\text { estromales endometriales. }\end{array}$ & $\begin{array}{c}\text { Valores de } p<0,01 \text { para los tres } \\
\text { antioxidantes }\end{array}$ \\
\hline $\begin{array}{l}\text { Rodrigues et al., } \\
2009 \text { (22) }\end{array}$ & Sangre & $\begin{array}{l}\text { Niveles de } \\
\text { Malonildialdehído } \\
\text { (MDA), vitamina } \\
\text { E, glutatión (GHS) } \\
\text { y capacidad } \\
\text { antioxidante. }\end{array}$ & $\begin{array}{l}\text { No se encontraron diferencias en los niveles } \\
\text { de MDA, vitamina E, GHS y capacidad } \\
\text { antioxidante entre el control y las mujeres } \\
\text { con endometriosis. }\end{array}$ & $\begin{array}{c}\text { MDA en Control }=0,0030 \pm \\
0,0009 \mathrm{mmol} / \mathrm{g} \\
\text { Endometriosis }=0,0033 \pm \\
0,0006 \mathrm{mmol} / \mathrm{g} \\
\text { Vitamina } \mathrm{E} \text { en Control }=21,7 \pm \\
\quad 6,3 \mathrm{mmol} / \mathrm{L} \\
\text { Endometriosis }=9,2 \pm 6,3 \mathrm{mmol} / \mathrm{L} \\
\text { Capacidad antioxidante } \\
\text { Control }=12,5 \pm 6,8 \text { eq trolox } \\
\text { Endometriosis }=9,6 \pm 2,3 \text { eq trolox }\end{array}$ \\
\hline $\begin{array}{l}\text { Lambrinoudaki } \\
\text { et al., } 2009 \text { (39) }\end{array}$ & $\begin{array}{l}\text { Sangre } \\
\text { Hsp70b, }\end{array}$ & $\begin{array}{l}\text { Niveles de Hsp70, } \\
\text { Tioredoxina (TRX), } \\
\text { albúmina } \\
\text { modificada por } \\
\text { isquemia (IMA). }\end{array}$ & $\begin{array}{l}\text { Las mujeres con endometriosis mostraron } \\
\text { niveles más altos de Hsp70b en suero } \\
\text { comparado con el grupo control. } \\
\text { El estadio de la endometriosis no afecta los } \\
\text { niveles Hsp70b. }\end{array}$ & $\begin{array}{c}\text { Hsp70b en Control }=0,135 \pm \\
0,014 \mathrm{ng} / \mathrm{mL} \\
\text { Endometriosis }=0,718 \pm 0,103 \mathrm{ng} / \mathrm{mL}\end{array}$ \\
\hline $\begin{array}{l}\text { Jackson et al., } \\
2005 \text { (40) }\end{array}$ & Sangre & $\begin{array}{l}\text { Niveles de } \\
\text { vitamina E. } \\
\text { Actividad } \\
\text { paraoxonasa. }\end{array}$ & $\begin{array}{l}\text { Las mujeres con endometriosis tienen } \\
\text { niveles significativamente más altos que } \\
\text { el grupo control. } \\
\text { La actividad paraoxonasa es más baja en } \\
\text { las mujeres con endometriosis. }\end{array}$ & $\begin{array}{l}\text { Vitamina } E \text { en Control y en } \\
\text { Endometriosis, } \mathrm{p}=0,04 \\
\text { Actividad paraoxonasa en } \\
\text { Control }=223,1 \mathrm{IU} / \mathrm{L} \\
\text { Endometriosis }=195,4 \mathrm{IU} / \mathrm{L} \\
\qquad p=0,09\end{array}$ \\
\hline $\begin{array}{l}\text { Jana et al., } \\
2012(29)\end{array}$ & $\begin{array}{l}\text { Modelo en } \\
\text { ratones }\end{array}$ & $\begin{array}{l}\text { Actividad MMP-3 } \\
\text { Expresión de } \\
\quad \text { NF-kB } \\
\text { Expresión de Bax, } \\
\text { BcL2, Citocromo C }\end{array}$ & $\begin{array}{l}\text { Este estudio sugiere que la Actividad MMP-3 } \\
\text { juega un rol importante en el desarrollo de } \\
\text { endometriosis animal y la progresión de la } \\
\text { enfermedad. La administración de curcumina } \\
\text { induce la regresión de la endometriosis } \\
\text { vía apoptótica mitocondrial. }\end{array}$ & No reportado \\
\hline $\begin{array}{l}\text { Murphy et al., } \\
1998 \text { (41) }\end{array}$ & $\begin{array}{l}\text { Biopsias de tejido } \\
\text { endometrial } \\
\text { eutópico y } \\
\text { ectópico }\end{array}$ & $\begin{array}{l}\text { Complejos de } \\
\text { lipoproteínas } \\
\text { oxidadas }\end{array}$ & $\begin{array}{l}\text { Los resultados encontrados sugieren que el } \\
\text { endometrio ectópico puede ser una fuente } \\
\text { de lipoproteínas oxidadas. }\end{array}$ & No reportado \\
\hline $\begin{array}{l}\text { Ota et al., } \\
2002(25)\end{array}$ & $\begin{array}{l}\text { Biopsias de } \\
\text { tejido } \\
\text { endometrial } \\
\text { eutópico y } \\
\text { ectópico }\end{array}$ & $\begin{array}{l}\text { Expresión de } \\
\text { catalasa (CAT) }\end{array}$ & $\begin{array}{l}\text { Los resultados evidenciaron una anormal } \\
\text { expresión de CAT en el tejido endometrial } \\
\text { eutópico y ectópico. }\end{array}$ & No reportado \\
\hline
\end{tabular}




\begin{tabular}{|c|c|c|c|c|}
\hline Autor & $\begin{array}{l}\text { Tipo de } \\
\text { muestra }\end{array}$ & Marcador & Hallazgo cualitativo & Hallazgo cuantitativo \\
\hline $\begin{array}{c}\text { Donabela et al., } \\
2010(42)\end{array}$ & Sangre & $\begin{array}{l}\text { Hidroperóxidos } \\
\text { lipídicos, productos } \\
\text { de oxidación } \\
\text { proteica avanzada } \\
\text { (AOPP) y } \\
\text { glutatión (GHS) }\end{array}$ & $\begin{array}{l}\text { Los niveles de AOPP fueron significativamente } \\
\text { altos en las mujeres con endometriosis } \\
\text { respecto al control. } \\
\text { Los niveles GHS fueron bajos respecto } \\
\text { al control. }\end{array}$ & $\begin{array}{c}\text { Niveles de AOPP }(\mu \mathrm{mol} / \mathrm{L}) \\
\text { en endometriosis: }(\mathrm{I} / \mathrm{II})=125,9 \pm 57,8 \\
(\mathrm{III} / \mathrm{IV})=137,6 \pm 57,6 \\
\text { Control }=97,0 \pm 55,8 p<0,05 \\
\text { GHS }(\mathrm{nMol} / \mathrm{L}) \text { en endometriosis: } \\
(\mathrm{I} / \mathrm{II})=125,9 \pm 57,8 \\
(\mathrm{III} / \mathrm{IV})=137,6 \pm 57,6 \\
\text { Control }=168,7 \pm 52,4 p<0,05\end{array}$ \\
\hline $\begin{array}{l}\text { Guney et al., } \\
2008 \text { (30) }\end{array}$ & $\begin{array}{l}\text { Explantes } \\
\text { endometriales } \\
\text { en un modelo de } \\
\text { endometriosis } \\
\text { en ratas }\end{array}$ & $\begin{array}{c}\text { Ciclooxigenasa-2 } \\
\text { (COX-2), } \\
\text { superóxido } \\
\text { dismutasa (SOD), } \\
\text { catalasa (CAT), } \\
\text { malonildialdehído } \\
\text { (MDA) }\end{array}$ & $\begin{array}{l}\text { Disminución del volumen del explante, } \\
\text { aumento en la actividad de CAT y SOD, } \\
\text { y disminución de MDA en ratas tratadas } \\
\text { con melatonina. }\end{array}$ & $\begin{array}{c}\text { Volumen antes tto }=141,4 \pm 34,3 \\
\text { Después tto }=42,9 \pm 14 \mu \mathrm{g} / \mathrm{mL} p<0,01 \\
\text { SOD antes tto }=59,63 \pm 15,8 \mathrm{U} / \mathrm{g} \text { prot } \\
\text { Después tto }=104 \pm 17,1 \mathrm{U} / \mathrm{g} \text { prot } \\
p<0,01 \\
\text { CATantes tto }=0,68 \pm 0,13 \mathrm{k} / \mathrm{g} \text { prot } \\
\text { Después tto }=1,25 \pm 0,18 \mathrm{k} / \mathrm{g} \text { prot } \\
\qquad<0,01 \\
\text { MDA antes tto }=4,7 \pm 0,33 \mathrm{nmol} / \mathrm{g} \text { prot } \\
\text { Después tto }=3,56 \pm 0,32 \mathrm{nmol} / \mathrm{g} \text { prot } \\
p<0,01\end{array}$ \\
\hline $\begin{array}{l}\text { Kyo et al., } \\
2010 \text { (43) }\end{array}$ & $\begin{array}{l}\text { Suero y fluido } \\
\text { peritoneal }\end{array}$ & $\begin{array}{c}\text { Niveles de } \\
\text { tioredoxina (TRX) } \\
\text { y tioredoxina } \\
\text { unida a proteína } 2 \\
\text { (TBP-2) }\end{array}$ & $\begin{array}{l}\text { No hay diferencias significativas en los niveles } \\
\text { de RNAm de TRX pero sí en ARNm TBP-2 } \\
\text { en pacientes con endometriosis vs el grupo } \\
\text { control, por lo que la relación TRX/TBP-2 } \\
\text { está aumentada en mujeres con endometriosis. }\end{array}$ & $\begin{array}{l}\text { TRX } / \text { TBP-2 }=4,5 \text { (endometriosis) } \\
\text { TRX } / \text { TBP-2 = 1,5 (control) }\end{array}$ \\
\hline $\begin{array}{l}\text { Ota et al., } \\
1999(25)\end{array}$ & $\begin{array}{l}\text { Tejido } \\
\text { endometrial } \\
\text { eutópico y } \\
\text { ectópico }\end{array}$ & $\begin{array}{c}\text { Expresión de } \\
\text { superóxido } \\
\text { dismutasa (SOD) }\end{array}$ & $\begin{array}{l}\text { Los resultados evidenciaron una anormal } \\
\text { expresión de SOD tanto en el tejido } \\
\text { endometrial eutópico y ectópico, } \\
\text { independiente del ciclo menstrual. }\end{array}$ & No reportado \\
\hline $\begin{array}{l}\text { Slater et al., } \\
2005(45)\end{array}$ & $\begin{array}{l}\text { Tejido } \\
\text { endometrial } \\
\text { eutópico y } \\
\text { ectópico }\end{array}$ & $\begin{array}{c}\text { Presencia de 8- } \\
\text { nosina (8-OHdG) }\end{array}$ & $\begin{array}{l}\text { Este estudio indica que las células } \\
\text { endometrióticas son anormales y exhiben } \\
\text { daño oxidativo al ADN, comparado } \\
\text { con el endometrio normal. }\end{array}$ & No reportado \\
\hline $\begin{array}{l}\text { Ngo et al., } \\
2005 \text { (27) }\end{array}$ & $\begin{array}{l}\text { Cultivo celular } \\
\text { primario células } \\
\text { endometriales. } \\
\text { Modelo animal }\end{array}$ & $\begin{array}{c}\text { Actividad } \\
\text { enzimática } \\
\text { de superóxido } \\
\text { dismutasa (SOD) } \\
\text { y ensayos de } \\
\text { proliferación } \\
\text { celular. }\end{array}$ & $\begin{array}{l}\text { Este trabajo demostró que las células } \\
\text { endometrióticas de pacientes con endometriosis } \\
\text { tienen alterado el fenotipo en la producción de } \\
\text { especies reactivas, lo cual lleva al incremento } \\
\text { de la capacidad de proliferación. }\end{array}$ & $\begin{array}{c}\text { Los incrementos de especies reactivas } \\
\text { tuvieron valores de } p<0,05 \text { en } \\
\text { células estromales y epiteliales } \\
\mathrm{N} \text {-acetilcisteina, } \mathrm{Ru}-486 \text {, danzol, reducen } \\
\text { la proliferación de células endometrióticas } \\
\text { en modelos animales } p<0,05\end{array}$ \\
\hline $\begin{array}{l}\text { Oner-Iyidogan } \\
\text { et al., } 2005 \text { (26) }\end{array}$ & $\begin{array}{l}\text { Tejido } \\
\text { endometrial } \\
\text { eutópico y } \\
\text { ectópico }\end{array}$ & $\begin{array}{l}\text { Actividad } \\
\text { superóxido } \\
\text { dismutasa (SOD). } \\
\text { Niveles de } \\
\text { malonildialdehído } \\
\text { (MDA). }\end{array}$ & $\begin{array}{l}\text { La actividad SOD en tejido endometrial } \\
\text { ectópico fue mayor que en el tejido } \\
\text { endometrial normal. } \\
\text { Los niveles de MDA fueron mayores en el } \\
\text { tejido endometrial ectópico. }\end{array}$ & $\begin{array}{l}\text { Actividad SOD en tejido ectópico } \\
=3512 \pm 1502 \mathrm{U} / \mathrm{g} \text { de prot } \\
\text { Tejido eutópico }=1893 \pm 780 \mathrm{U} / \mathrm{g} \\
\text { de prot } p=0,002 \\
\text { Niveles de } \mathrm{MDA} \text { en tejido ectópico } \\
=206,26 \pm 78,70 \mathrm{nmol} / \mathrm{g} \text { de prot } \\
\text { Tejido eutópico }=160,28 \pm 2074 \\
\mathrm{nmol} / \mathrm{g} \text { de prot }\end{array}$ \\
\hline
\end{tabular}




\begin{tabular}{|c|c|c|c|c|}
\hline Autor & $\begin{array}{l}\text { Tipo de } \\
\text { muestra }\end{array}$ & Marcador & Hallazgo cualitativo & Hallazgo cuantitativo \\
\hline $\begin{array}{l}\text { Polack et al., } \\
2001(42)\end{array}$ & $\begin{array}{l}\text { Líquido } \\
\text { peritoneal }\end{array}$ & $\begin{array}{l}\text { Estado total } \\
\text { antioxidante }\end{array}$ & $\begin{array}{l}\text { No se encontraron diferencias en el estado } \\
\text { total antioxidante de mujeres con } \\
\text { endometriosis vs mujeres infértiles. }\end{array}$ & $\begin{array}{l}\text { Estado total antioxidante en infértiles } \\
\qquad \begin{array}{c}=0,496 \pm 0,21 \mathrm{nmol} / \mathrm{L} \\
\text { endometriosis }=0,616 \pm 0,2 \mathrm{nmol} / \mathrm{L} \\
\qquad p>0,05\end{array}\end{array}$ \\
\hline $\begin{array}{l}\text { Polack et al., } \\
2003(43)\end{array}$ & $\begin{array}{l}\text { Líquido } \\
\text { peritoneal }\end{array}$ & $\begin{array}{c}\text { Niveles de } \\
\text { glutatión (GHS) }\end{array}$ & $\begin{array}{l}\text { No hay diferencias en los niveles de glutatión } \\
\text { de las mujeres con endometriosis vs el control. }\end{array}$ & $\begin{array}{l}\text { Niveles de GHS en mujeres con estadio } \mathrm{I} / \mathrm{II} \\
\text { de endometriosis }=0,22 \pm 0,01 \mu \mathrm{mol} / \mathrm{mL} \\
\text { Mujeres con estadio III/IV de endometriosis } \\
\quad=0,24 \pm 0,03 \mu \mathrm{mol} / \mathrm{mL} \\
\text { Grupo control }=0,23 \pm 0,0503 \mu \mathrm{mol} / \mathrm{mL}\end{array}$ \\
\hline $\begin{array}{l}\text { Pasqualotto } \\
\text { et al., } 2004(23)\end{array}$ & Fluido folicular & $\begin{array}{l}\text { Capacidad } \\
\text { antioxidante total } \\
\text { y niveles de } \\
\text { malonildialdehído } \\
\text { (MDA) }\end{array}$ & $\begin{array}{l}\text { La capacidad antioxidante fue mayor } \\
\text { en las mujeres embarazadas. } \\
\text { Los niveles de MDA fueron mayores en } \\
\text { las mujeres no embarazadas. }\end{array}$ & $\begin{array}{c}\text { Capacidad antioxidante } \\
\rightarrow 819,16 \text { eq de Trolox } \\
\text { Niveles de } \mathrm{MDA}=0,95 \mu \mathrm{mol} \text { de } \mathrm{MDA} / \mathrm{L}\end{array}$ \\
\hline $\begin{array}{l}\text { Do Amaral et al., } \\
2005 \text { (24) }\end{array}$ & $\begin{array}{l}\text { Líquido } \\
\text { peritoneal }\end{array}$ & $\begin{array}{l}\text { Niveles de } \\
\text { malonildialdehído } \\
\text { MDA, colesten } \\
\text {-3,5-dien-7-ona } \\
\text { (CSD) }\end{array}$ & $\begin{array}{l}\text { No hay diferencias entre el grupo control vs } \\
\text { mujeres con endometriosis en lo que respecta } \\
\text { a los niveles de MDA y CSD. }\end{array}$ & $\begin{array}{l}\text { Niveles de MDA/MDA Cu} \\
\text { endometriosis }=0,07 \text { y } 0,34 \mathrm{nmodiana}) \text { en } / \mathrm{mL} \\
\text { Grupo control }=0,04 \text { y } 0,21 \mathrm{nmol} / \mathrm{mL} \\
\qquad P=0,09 \text { y } 0,5 \\
\text { Niveles de CSD (mediana) en con } \\
\text { endometriosis }=0,24 \mu \mathrm{g} / \mathrm{mL} \\
\text { Grupo control }=0,25 \mu \mathrm{g} / \mathrm{mL}\end{array}$ \\
\hline $\begin{array}{l}\text { Appasamy et al., } \\
2008 \text { (37) }\end{array}$ & $\begin{array}{c}\text { Plasma } \\
\text { fluido folicular }\end{array}$ & $\begin{array}{l}\text { Capacidad } \\
\text { antioxidante } \\
\text { total (TCA) }\end{array}$ & $\begin{array}{l}\text { Se encontró una asociación significativamente } \\
\text { fuerte entre los niveles de TCA en plasma } \\
\text { y fluido folicular. }\end{array}$ & Asociación positiva $\mathrm{r}=0,45 \mathrm{y} p<, 001$ \\
\hline $\begin{array}{l}\text { Kyama et al., } \\
2008 \text { (36) }\end{array}$ & $\begin{array}{l}\text { Tejido } \\
\text { endometrial y } \\
\text { tejido peritoneal }\end{array}$ & $\begin{array}{l}\text { Expresión de ARN } \\
\text { de aromatasa, } \\
\text { citoquinas y factores } \\
\text { de adhesión }\end{array}$ & $\begin{array}{l}\text { El incremento de la expresión de citoquinas } \\
\text { inflamatorias, aromatasa y factores de } \\
\text { adhesión en el endometrio y peritoneo } \\
\text { normal de pacientes con endometriosis sugiere } \\
\text { que ambos tejidos son afectados por las fases } \\
\text { del ciclo menstrual o la presencia o ausencia } \\
\text { de endometriosis. }\end{array}$ & $p<0,05$ \\
\hline $\begin{array}{c}\text { Shanti et al., } \\
1999 \text { (46) }\end{array}$ & $\begin{array}{l}\text { Sangre } \\
\text { líquido } \\
\text { peritoneal }\end{array}$ & $\begin{array}{c}\text { Niveles de } \\
\text { autoanticuerpos } \\
\text { para LDL } \\
\text {-malonildialdehído } \\
\text { modificada } \\
\text { oxidativamente, } \\
\text { LDL oxidada } \\
\text { (LDL ox) }\end{array}$ & $\begin{array}{l}\text { Los resultados muestran que el incremento de } \\
\text { autoanticuerpos de estrés oxidativo en } \\
\text { mujeres con endometriosis soporta la } \\
\text { hipótesis de la relación entre estrés oxidativo } \\
\text { y endometriosis. }\end{array}$ & $\begin{array}{c}\text { Niveles MDA (D.O) en endometriosis } \\
=0,216 \pm 0,005 \\
\text { Control }=0,16 \pm 0,003 \\
p<0,0005 \\
\text { Niveles de LDL-ox (D.O) en endometriosis } \\
=0,22 \pm 0,005 \\
\text { Control }=0,18 \pm 0,006, p<0,04)\end{array}$ \\
\hline $\begin{array}{l}\text { Song et al., } \\
2004(46)\end{array}$ & $\begin{array}{l}\text { Tejido } \\
\text { endometrial } \\
\text { eutópico }\end{array}$ & Niveles de $\mathrm{BCl}_{2}$ & $\begin{array}{l}\text { La administración de vitaminas } \mathrm{E} \text { y } \mathrm{C} \text { decrece } \\
\text { los niveles de } \mathrm{Bcl}_{2} \text { en el tejido endometrial de } \\
\text { mujeres con endometriosis. }\end{array}$ & $\begin{array}{l}\text { Niveles BcL2 en endometriosis } \\
\qquad=3,13 \pm 1,03 \\
\text { Endometriosis y suplementación con } \\
\text { vitamina } \mathrm{E} \text { y } \mathrm{C}=2,17 \pm 0,44 \\
\qquad p<0,04\end{array}$ \\
\hline $\begin{array}{l}\text { Romao et al., } \\
2009 \text { (19) }\end{array}$ & Sangre & $\begin{array}{c}\text { Niveles de } \\
\text { malonildialdehído } \\
\text { (MDA), } \\
\text { vitamina E, } \\
\text { glutatión (GHS) } \\
\text { y capacidad } \\
\text { antioxidante total } \\
\text { (TAC) }\end{array}$ & $\begin{array}{l}\text { Los niveles en suero de GHS pueden estar } \\
\text { correlacionados inversamente con los estadios } \\
\text { de la endometriosis. }\end{array}$ & $\begin{array}{l}\text { Niveles de GHS } \geq 137,8 \text { es predictor de } \\
\text { moderada o severa endometriosis } \\
\text { (estadio } 3 \text { o } 4 \text { ) con } 93,7 \% \text { de sensibilidad, } \\
54,5 \% \text { de especificidad y } 79 \% \text { de exactitud. }\end{array}$ \\
\hline
\end{tabular}




\begin{tabular}{|c|c|c|c|c|}
\hline Autor & $\begin{array}{l}\text { Tipo de } \\
\text { muestra }\end{array}$ & Marcador & Hallazgo cualitativo & Hallazgo cuantitativo \\
\hline $\begin{array}{c}\text { Prieto et al., } \\
2012 \text { (17) }\end{array}$ & $\begin{array}{c}\text { Plasma y } \\
\text { fluido folicular }\end{array}$ & $\begin{array}{l}\text { Concentraciones } \\
\text { de vitamina C, } \\
\text { vitamina } \mathrm{E}, \\
\text { malonildialdehído } \\
\text { (MDA) y } \\
\text { superóxido } \\
\text { dismutasa (SOD) }\end{array}$ & $\begin{array}{l}\text { Mujeres con endometriosis mostraron bajas } \\
\text { concentraciones de vitamina C en fluido } \\
\text { folicular y SOD en plasma, altos niveles de } \\
\text { vitamina E, sin diferencias en las } \\
\text { concentraciones de malonildialdehído cuando } \\
\text { son comparadas con el grupo control, } \\
\text { sugiriendo que estas tienen baja } \\
\text { capacidad antioxidante. }\end{array}$ & $\begin{array}{c}\text { Vitamina } C \text { en endometriosis } \\
=12,7 \pm 5,9 \mu \mathrm{g} / \mathrm{mL} \\
\text { Control }=9,7 \pm 6,9 \mu \mathrm{g} / \mathrm{mL} \\
p=0,003 \\
\text { SOD en endometriosis }=0,9 \pm 1,4 \mathrm{U} / \mathrm{mL} \\
\text { Control }=0,55 \pm 0,7 \mathrm{U} / \mathrm{mL} \\
p=0,059 \\
\text { Vitamina E en endometriosis } \\
=8,1 \pm 3,8 \mu \mathrm{g} / \mathrm{mL} \\
\text { Control }=5,2 \pm 3,2 \mu \mathrm{g} / \mathrm{mL} \\
p=0,001 \\
\text { MDA en endometriosis } \\
=57,6 \pm 44,2 \mu \mathrm{g} / \mathrm{mL} \\
\text { Control }=46 \pm 29,1 \mu \mathrm{g} / \mathrm{mL} \\
\text { p no reportado }\end{array}$ \\
\hline
\end{tabular}

señala la ausencia de uniformidad en los resultados obtenidos para precisar sobre la real asociación entre estrés oxidativo y endometriosis pélvica.

A partir de los conceptos básicos conocidos de interacción química y biología en las células, tejidos y fluidos, la búsqueda de marcadores de estrés oxidativo, prooxidantes o antioxidantes ofrece un campo de investigación para la búsqueda de marcadores bioquímicos que puedan favorecer el diagnóstico temprano y el seguimiento de las mujeres con endometriosis (1). Además, los estudios in vitro pueden permitir el origen de agentes terapéuticos antioxidantes específicos que influyan sobre la evolución de la enfermedad con el fin de mejorar la calidad de vida de las mujeres que la padecen. La bioquímica ofrece un amplio campo de investigación para adelantar los estudios que se ameritan a fin de develar los aspectos etiológicos involucrados en el establecimiento y la progresión de la endometriosis, aun de causa desconocida e identificada como la enfermedad de las teorías (2).

\section{CONCLUSIÓN}

No hay estudios amplios y sólidos que permitan aseverar la asociación entre estrés oxidativo y endometriosis. Esta asociación ofrece un campo por explorar para la investigación básica y transnacional.

\section{REFERENCIAS}

1. Giudice L. Endometriosis. NEJM. 2010;362:2389-98.

2. Vinatier D, Orazi G, Cosson M, Dufour P. Theories of endometriosis. Eur J Obstet Gynecol Reprod Biol. 2001;96:21-34.

3. Kavtaradze N, Dominguez C, Rock J, Parthasarathy S, Murphy A. Vitamin E and C supplementation reduces endometriosis related pelvic pain. Fertil Steril. 2003;80:S221-2.

4. Langendonckt AV, Casanas-Roux F, Donnez J. Oxidative stress and peritoneal endometriosis. Fertil Steril. 2002;77:861-70.

5. Sagrista M, Garcia A, Africa de Madariaga M, Mora M. Antioxidant and pro-oxidant effect of thiolic compounds N-acetyl-L-cysteine and glutathione against free radical-induced lipid peroxidation. Free Rad Res. 2002;36:329-40.

6. Shackerford R, Kaufmann W, Paules R. Oxidative stress and cell cycle checkpoint function. Free Radic Biol Med. 2000;28:1387-1404.

7. Goldstein S, Meyerstein D, Czapski G. The Fenton Reagents. Free Radic Biol Med. 1993;15:435-45.

8. Halliwell B, Gutteridge M. Role of free radicals and catalytic metal ions. In human disease: An overview. Meth Enzymol. 1990;186:1-85.

9. Liu M, Pelling J, Ju J, Chu E, Brash D. Antioxidant action via p5-mediated apoptosis. Cancer Res. 1998;58:1723-9. 
10. Mates J, Sánchez F. Role of reactive oxygen species in apoptosis: implications for cancer therapy. Internat J Biochem Cell Biol. 2000;32:157-70.

11. Dreher D, Junod A. Role of oxygen free radicals in cancer development. Eur J Cancer. 1996;32:30-8.

12. Peeters-Joris C, Vandevoorde A, Baudhuin P. Subcellular Localization of Superoxide Dismutase in Rat Liver. Biochem. J 1975;150:31-9.

13. Seeber BE, Czech T, Buchner H, Barnhart KT, Seger Ch, Daxenbichler G, et al. The vitamin E-binding protein afamin is altered signifcantly in the peritoneal fluid of women with endometriosis. Fertil Steril. 2010;94:2923-6.

14. Savaris AL, Amaral VF. Nutrient intake, anthropometric data and correlations with the systemic antioxidant capacity of women with pelvic endometriosis. Eur J Obstet Gynecol Reprod Biol. 2011;158:314-8.

15. Yoon S, Yun C, Chung A. Dose effect of oxidative stress on signal transduction in aging. Mechan Ageing Develop. 2002;123:1597-604.

16. Lousse J, Defrere S, Langendonckt A, Gras J, Gonzalez $\mathrm{R}$, Colette $\mathrm{S}$, et al. Iron storage is significantly increased in peritoneal macrophages of endometriosis patients and correlates with iron overload in peritoneal fluid. Fertil Steril. 2009;91:1668-75.

17. Prieto L, Quesada J, Cambero O, Pacheco A, Pellicer A, Codoceo R, et al. Analysis of follicular fluid and serum markers of oxidative stress in women with infertility related to endometriosis. Fertil Steril. 2012;98:126-30.

18. Campos C, Ferriani R, Dos Reis R, Dias De Moura M, Jordão A, De Albuquerque P. Lipid peroxidation and vitamin $\mathrm{E}$ in serum and follicular fluid of infertile women with peritoneal endometriosis submitted to controlled ovarian hyperstimulation: a pilot study. Fertil Steril. 2008;90:2080-5.

19. Song M, Dominguez C, Lowe E, Parthasarathy S, Murphy AA. Antioxidants (vitamins E and C) decrease $\mathrm{Bcl} 2 /$ increase apoptosis in eutopic endometrium of women with endometriosis. Fertil Steril. 2004;82:S166-S167.

20. Szczepanska M, Kozlik J, Skrzypczak J, Mikołajczyk M. Oxidative stress may be a piece in the endometriosis puzzle. Fertil Steril. 2003;79:1288-93.
21. Mier-Cabrera J, Genera M, De la Jara-Díaz J, PerichartPerera O, Vadillo F, Hernández C. Effect of vitamins C and E supplementation on peripheral oxidative stress markers and pregnancy rate in women with endometriosis. Internat J Gynecol Obstet. 2008;100:252-6.

22. Rodrigues J, Dib L, Melo A, Cardoso R, Romao G, Navarro P. Analysis of serum markers of oxidative stress response in women with infertility related to endometriosis. Fertil Steril. 2009;92:S113.

23. Pasqualotto E, Agarwal A, Sharma R, Izzo V, Pinnotti J, Joshi N, et al. Effect of oxidative stress in follicular fluid on the outcome of assisted reproductive procedures. Fertil Steril. 2004;81:973-6.

24. Do Amaral, Bydlowski S, Peranovich T, Navarro P, Subbiahb M, Ferriani R. Lipid peroxidation in the peritoneal fluid of infertile women with peritoneal endometriosis. Eur J Obstet Gynecol Reprod Biol. 2005;119:72-5.

25. Ota H, Igarashi S, Sato N, Tanaka H, Tanaka T. Involvement of catalase in the endometriosis and adenomyosis. Fertil Steril. 2002;78:804-9.

26. Oner-Iyidogan Y, Kocak H, Gurdol F, Korkmaz D, Buyru F. Indices of oxidative stress in eutopic and ectopic endometria of women with endometriosis. Gynecol Obstet Invest. 2004;57:214-7.

27. Ngo C, Chereau C, Nicco C, Weill B, Chapron C, Batteux F. Reactive oxygen species controls endometriosis progression. Am J Pathol. 2009;175:225-34.

28. Ota H, Igarashi S, Hatazawa J, Tanaka T. Immunohistochemical assessment of superoxide dismutase expression in the endometrium in endometriosis and adenomyosis. Fertil Steril. 1999;72:129-34.

29. Jana S, Paul S, Swarnakar S. Curcumin as antiendometriotic agent: Implication of MMP-3 and intrinsic apoptotic pathway. Biochem Pharmacol. 2012;83:797-804

30. Güney M, Oral B, Karahan N, Mungan T. Regression of endometrial explants in rat model of endometriosis treated with melatonin. Fertil Steril. 2008;89:934-42.

31. Foyouzi N, Berkkanoglu M, Arici A, Kwintkiewicz J, Izquierdo D, Duleba AJ. Effects of oxidants and antioxidants on proliferation of endometrial stromal cells. Fertil Steril. 2004;82:S1019-22. 
32. Agarwal A, Cocuzza M, Abdelrazik H, Sharma R. Oxidative stress measurement with male or female factor infertility. Handbook of Chemiluminescent Methods in Oxidative Stress Assessment; 2008. p. 195-218.

33. Pejic S, Todorovic A, Stojiljkovic V, Cvetkovic D, Lucic N, Radojicic R, et al. Superoxide dismutase and lipid hydroperoxides in blood and endometrial tissue of patients with benign, hyperplastic and malignant endometrium. Annals Brazil Acad Sci. 2008;80:515-22.

34. Carvalho L, Podgaec S, Bellodi-Privato M, Falcone T, Simoes M. Role of eutopic endometrium in pelvic Endometriosis. J Minim Inv Gynecol. 2011;18:419-27.

35. Polack G, Wertel I, Koziol-Montewka M, Tarkowski R, Kotarski J. Investigation of glutathione concentrations in peritoneal fluid from women with and without endometriosis. Eur J Obstet Gynecol Reprod Biol. 2003;109:206-8.

36. Kyama CM, Overbergh L, Mihalyi A, Meuleman C, Mwenda JM, Mathieu C, et al. Endometrial and peritoneal expression of aromatase cytokines and adhesion factors in women with endometriosis. Fertil Steril. 2008;89:301-10.

37. Appasamy M, Jauniaux E, Serhal P, Al-Qahtani A, Groome N, Muttukrishna S. Evaluation of the relationship between folicular fluid oxidative stress, ovarían hormones, and response to gonadotropin stimulation. Fertil Steril. 2008;89:912-21.

38. Azevedo A, Ormanji M, FraiettaR, DeFreitas V, Agarwal A, Bertoncini C. Detection of the oxidative stress levels in patients with and without endometriosis by analysis of confocal microscopy images using a superoxide probe. Fertil Steril. 2010;94:S203.

39. Lambrinoudaki I, Augoulea A, Christodoulakos G, Economou E, Kaparos G, Kontoravdis A, et al. Measurable serum markers of oxidative stress response in women with endometriosis. Fertil Steril. 2009;91:46-50.

40. Jackson L, Schisterman E, Dey-Rao R, Browne R, Armstrong D. Oxidative stress and endometriosis. Hum Reprod. 2005;20:2014-20.

41. Murphy A, Palinski W, Rankin S, Morales A, Parthasarathy S. Evidence for oxidatively modified lipid-protein complexes in endometrium and endometriosis. Fertil Steril. 1998;69:1092-4.

42. Donabela F, Andrade A, Rodrigues J, Dib L, Jordao A, Navarro P. Serum markers of oxidative stress in infertile women with endometriosis and controls. Fertil Steril. 2010;94:S40.

43. Kyo S, In Yang H, Eun K, Yeon H, Cho S, Sik Y, et al. The roles of thioredoxin and thioredoxinbinding protein-2 in endometriosis. Hum Reprod. 2010;25:1251-8.

44. Slater M, Quagliotto G, Cooper M, Murphy C. Endometriotic cells exhibit metaplastic change and oxidative DNA damage as well as decreased function, compared to normal endometrium. J Mol Histol. 2005;36:257-63.

45. Polack G, Koziol-Montewka M, Gogacz M, Blaszkowska I, Kotarski J. Total antioxidant status of peritoneal fluid in infertile women. Eur J Obstet Gynecol Reprod Biol. 2001;94:261-3.

46. Shanti A, Santanam N, Morales A, Parthasarathy S, Murphy A. Autoantibodies to markers of oxidative stress are elevated in women with endometriosis. Fertil Steril. 1999;71:1115-8.

47. Romao G, Zyman A, Rodrigues J, Dib L, Ferriani R, Navarro P. Relationship between serum markers of oxidative stress and endometriosis stage in endometriosis-associated infertility women. Fertil Steril. 2009;92:S221. 